\title{
As dificuldades encontradas na assistência à saúde às pessoas com surdez
}

\section{The difficulties encountered in health care for people with deafness}

\section{Las dificultades encontradas en la atención de la salud de las personas con sordera}

\author{
Marciele de Lima Silva \\ ORCID: https://orcid.org/0000-0003-2827-5316 \\ Instituto de Educação Superior da Paraíba, Brasil \\ E-mail: marcieledelsilva@gmail.com \\ Mariana Pereira Barbosa Silva \\ ORCID: https://orcid.org/0000-0003-0852-8099 \\ Universidade Estadual do Piauí, Brasil \\ E-mail: marianapbsilvaa@gmail.com \\ Airton César Leite \\ ORCID: https://orcid.org/0000-0001-7184-8488 \\ Centro Universitário Santo Agostinho, Brasil \\ E-mail: ainton.cesar2014@gmail.com \\ Beatriz Cardoso Melo \\ ORCID: https://orcid.org/0000-0002-3638-8815 \\ Centro Universitário Uninovafapi, Brasil \\ E-mail: beatrizcmelo@outlook.com \\ Allan Bruno Alves de Sousa Santos \\ ORCID: https://orcid.org/0000-0001-6412-7164 \\ Faculdade de Educação São Francisco, Brasil \\ E-mail: abass@faesf.com.br \\ Layanne Cavalcante de Moura \\ ORCID: https://orcid.org/0000-0003-2781-1076 \\ Centro Universitário UNIFACID, Brasil \\ E-mail: layannecavalcante@hotmail.com \\ Weberton Dorásio Sobrinho \\ ORCID: https://orcid.org/0000-0003-0619-7214 \\ Universidade de Rio Verde, Brasil \\ E-mail: dorasioweberton@gmail.com \\ Adson Gomes dos Santos \\ ORCID: https://orcid.org/0000-0002-3230-6165 \\ Universidade do Estado do Rio Grande do Norte, Brasil \\ E-mail: adsongomes@live.com \\ Ramires dos Santos Moraes \\ ORCID: https://orcid.org/0000-0002-8324-5219 \\ Centro Universitário UNIFACID, Brasil \\ E-mail: ramiresmoraes16@gmail.com \\ Gustavo Baroni Araujo \\ ORCID: https://orcid.org/0000-0002-3162-7477 \\ Universidade Estadual de Londrina, Brasil \\ E-mail: gustavobaroni13@hotmail.com \\ Angela Palma Amorim \\ ORCID: https://orcid.org/0000-0003-0594-795X \\ Centro Universitário da Amazônia, Brasil \\ E-mail: angelaamorim984@gmail.com \\ Joelma Maria dos Santos da Silva Apolinário \\ ORCID: https://orcid.org/0000-0001-9521-9432 \\ Faculdade Maurício de Nassau, Brasil \\ E-mail: jo.silva00@hotmail.com \\ Guilia Rivele Souza Fagundes \\ ORCID: https://orcid.org/0000-0003-1834-8278 \\ Universidade do Estado da Bahia, Brasil \\ E-mail: guilia_matina@ hotmail.com
}

\section{Resumo}

Este estudo tem como objetivo discutir a partir de achados na literatura as dificuldades encontradas na assistência à saúde às pessoas com surdez. Trata-se de um estudo descritivo, do tipo revisão integrativa de literatura, de caráter qualitativo, a busca ocorreu nas bases de dados: LILACS, SCIELO e BDENF, via BVS, e Scholar Google. Os artigos foram coletados no período de janeiro de 2021. Foram utilizados os descritores: Assistência à saúde, Surdez e Saúde 
pública, localizados na lista dos Descritores em Ciências da Saúde (DECs). Após a aplicação dos critérios de elegibilidade, restaram 14 estudos. A principal dificuldade na assistência a pessoa surda é a barreira comunicacional, devido à falta do conhecimento do profissional da saúde quanto a língua brasileira de sinais. Além disso a ausência de intérprete profissional nas instituições, discriminação, estereótipos. Conclui-se que é necessário profissionais capacitados a atender pacientes surdos para assim garantir uma assistência humanitária, integral e livre de preconceitos.

Palavras-chave: Assistência à saúde; Surdez; Saúde pública.

\begin{abstract}
This study aims to discuss, based on findings in the literature, the difficulties encountered in health care for people with deafness. It is a descriptive study, of the type integrative literature review, of a qualitative character, the search occurred in the databases: LILACS, SCIELO and BDENF, via VHL, and Scholar Google. The articles were collected in the period of January 2021. The descriptors: Health care, Deafness and public health were used, located in the list of Health Sciences Descriptors (DECs). After applying the eligibility criteria, 14 studies remained. The main difficulty in assisting the deaf is the communication barrier, due to the lack of knowledge of the health professional about the Brazilian sign language. In addition, the absence of a professional interpreter in the institutions, discrimination, stereotypes. It is concluded that it is necessary to have trained professionals to attend deaf patients in order to guarantee humanitarian assistance, comprehensive and free from prejudice.
\end{abstract}

Keywords: Health care; Deafness; Public health.

\title{
Resumen
}

Este estudio tiene como objetivo discutir, con base en los hallazgos de la literatura, las dificultades encontradas en la atención de la salud de las personas con sordera. Se trata de un estudio descriptivo, del tipo revisión integradora de la literatura, de carácter cualitativo, la búsqueda se realizó en las bases de datos: LILACS, SCIELO y BDENF, vía BVS, y Scholar Google. Los artículos fueron recolectados en el período de enero de 2021. Se utilizaron los descriptores: Atención de salud, Sordera y salud pública, ubicados en la lista de Descriptores de Ciencias de la Salud (DECs). Después de aplicar los criterios de elegibilidad, quedaron 14 estudios. La principal dificultad para ayudar a los sordos es la barrera de comunicación, debido a la falta de conocimiento del profesional de la salud sobre la lengua de signos brasileña. Además, la ausencia de un intérprete profesional en las instituciones, discriminación, estereotipos. Se concluye que es necesario contar con profesionales capacitados para atender a los pacientes sordos a fin de garantizar una asistencia humanitaria, integral y libre de prejuicios.

Palabras clave: Atención de la salud; Sordera; Salud pública.

\section{Introdução}

A audição é um dos sentidos mais nobres, uma vez que sua principal função está ligada à aquisição e ao desenvolvimento da linguagem oral, fundamental nas relações interpessoais e com o meio ambiente (Dantas et al., 2014).

No Brasil existe cerca de 45 milhões de pessoas portadoras de deficiência, destes 1,7 milhão de pessoas têm dificuldade em ouvir. Segundo padrões estabelecidos pela American National Standards Institute (ANSI) deficiência auditiva é definida como a diferença entre o desempenho do indivíduo e a habilidade normal para detecção sonora, o que gera dificuldade ou ausência em perceber os sons (Lessa \& Andrade, 2016).

A surdez pode ser classificada de três formas: bilateral, parcial ou total (podendo ser chamada também de severa ou profunda). Indivíduos com surdez severa podem apresentar perda total da audição, o que dificulta a interação social e a comunicação (França, Pontes, Costa \& França, 2016).

A deficiência auditiva é compreendida como um impedimento de natureza física, intelectual ou sensorial que, juntamente com diversas barreiras, pode dificultar a participação de um indivíduo na sociedade (Dantas et al., 2014).

A Lei de Acessibilidade ${ }^{\circ}$ 10.098, de 2000, atribuiu, como responsabilidade do poder público, o dever de promover a eliminação de barreiras na comunicação e estabelecer mecanismos e alternativas técnicas que torne os sistemas de comunicação acessíveis às pessoas com deficiência sensoriais (Brasil, 2000).

A libras foi reconhecida como meio legal da comunidade surda pela Lei Federal $n^{\circ} 10.436 / 02$ e regulamentada pelo Decreto $\mathrm{n}^{\circ}$ 5.626, 22 de dezembro de 2005. No art. 25 da mesma especifica que o serviço público de saúde deve garantir atendimento e tratamento adequado aos surdos e as instituições devem apoiar a capacitação e formação de profissionais para o 
uso de libras e sua tradução e interpretação (Brasil, 2002; Brasil, 2005). Porém, mesmo com conquistas legais, essa população ainda sofre por causa das barreiras comunicacionais, falta de informação e preconceito (Yonemotu \& Vieira, 2020).

A Lei $n^{\circ} 10.436$ de 24 de Abril de 2002, em seu art. 3º dispõe sobre a Língua Brasileira de Sinais (libras) e dá outras providências, nela consta que as instituições públicas e empresas concessionárias de serviços públicos de assistência à saúde devem garantir atendimento adequado aos portadores de deficiência auditiva (Brasil, 2002).

A assistência à pessoa deve ser baseada nos princípios de integralidade, equidade e universalidade, conforme previsto na Constituição Federal de 1988 e na Lei no 8.080/90. Sendo assim, não deve haver diferenciação no atendimento que prejudique a comunidade surda (Pereira, Passarin, Nishida, \& Garcez, 2020).

Por muito tempo as pessoas surdas eram conhecidas como doentes, com limitações e déficit cognitivo, estigmatizadas e alvo de preconceito. Atualmente, a sociedade compreende estes sujeitos como normais, apresentando habilidades, apesar disso os indivíduos ainda enfrentam muitos obstáculos. No Brasil é precária a acessibilidade dos mesmos aos serviços básicos, impedimento assim o acesso à saúde (Aragão et al., 2015).

Através de comunicação estabelecida com o paciente, o profissional pode compreendê-lo como ser integral e também perceber sua visão de mundo. É fundamental que o profissional estabeleça uma comunicação efetiva, para evitar erros na assistência (Cavagna, Silva, Braga \& Andrade, 2017).

A partir do momento em que existe uma falha de comunicação, pode ocorrer equívocos no diagnóstico, dificuldade em elaborar o prontuário e o tratamento inadequado para a determinada patologia, o acolhimento nos serviços de saúde é fundamental na garantia do direito à saúde (Soares, Lima, Santos \& Ferreira, 2018).

Os serviços de saúde brasileiros são responsáveis pelo atendimento à população de forma integral, guiados pelos programas do Ministério da Saúde com ações promotoras, preventivas e tratadoras da saúde dos indivíduos (Saraiva et al., 2017).

O despreparo dos profissionais prejudica a qualidade da assistência e a realização de um tratamento adequado. É fundamental que os profissionais da saúde conheçam a libras, para que possa compreender as pessoas com surdez e não comprometer a assistência prestada (Aragão et al., 2015).

Este estudo tem como objetivo discutir a partir de achados na literatura as dificuldades encontradas na assistência à saúde às pessoas com surdez.

\section{Metodologia}

Trata-se de um estudo descritivo, do tipo revisão integrativa de literatura, de caráter qualitativo. Segundo Mendes, Silveira, \& Galvão (2008) esse tipo de revisão inclui a análise de pesquisas relevantes que dão suporte para a tomada de decisão e a melhoria da prática clínica, permitindo a síntese do conhecimento de um determinado assunto, além disso, permite a síntese de múltiplos estudos publicados possibilitando conclusões gerais a respeito de uma área de estudo específica.

Foi seguida as seguintes etapas: definição do tema e elaboração da questão de pesquisa; elaboração dos critérios de elegibilidade, inclusão e exclusão dos estudos; definição dos descritores, busca na literatura e coleta de dados; análise crítica dos estudos incluídos e discussão dos resultados; e apresentação da síntese da revisão.

Para direcionar a presente revisão delineou-se como questão norteadora: "O que a literatura aborda sobre as dificuldades encontradas na assistência à saúde às pessoas surdas?".

Para a construção deste trabalho, a busca dos artigos foi realizada na Biblioteca Virtual em Saúde (BVS), com o auxílio das bases de dados Literatura Latino-Americana e do Caribe em Ciências da Saúde (LILACS), Scientific Electronic Library Online (SciELO), Base de Dados de Enfermagem (BDENF) e Scholar Google. Os artigos foram coletados no período 
de dezembro de 2020 a janeiro de 2021. Foram utilizados os descritores: "Assistência à saúde" AND "Surdez" AND "Saúde pública”, cruzados com o operador booleando "AND”, localizados na lista dos Descritores em Ciências da Saúde (DECs).

Foram selecionados como critérios de inclusão: artigos completos disponíveis na íntegra, nos idiomas português, espanhol e inglês, com recorte temporal de 2015 a 2020. E os critérios de exclusão foram: artigos incompletos, duplicados, debates, resenhas, resumos ou artigos publicados em anais de eventos, indisponíveis na íntegra.

\section{Resultados e Discussão}

O presente fluxograma representado abaixo caracteriza a estratégia de coleta de dados utilizada pelos autores, e busca detalhar as principais etapas da pesquisa.

Figura 1. Fluxograma de identificação e seleção dos artigos.

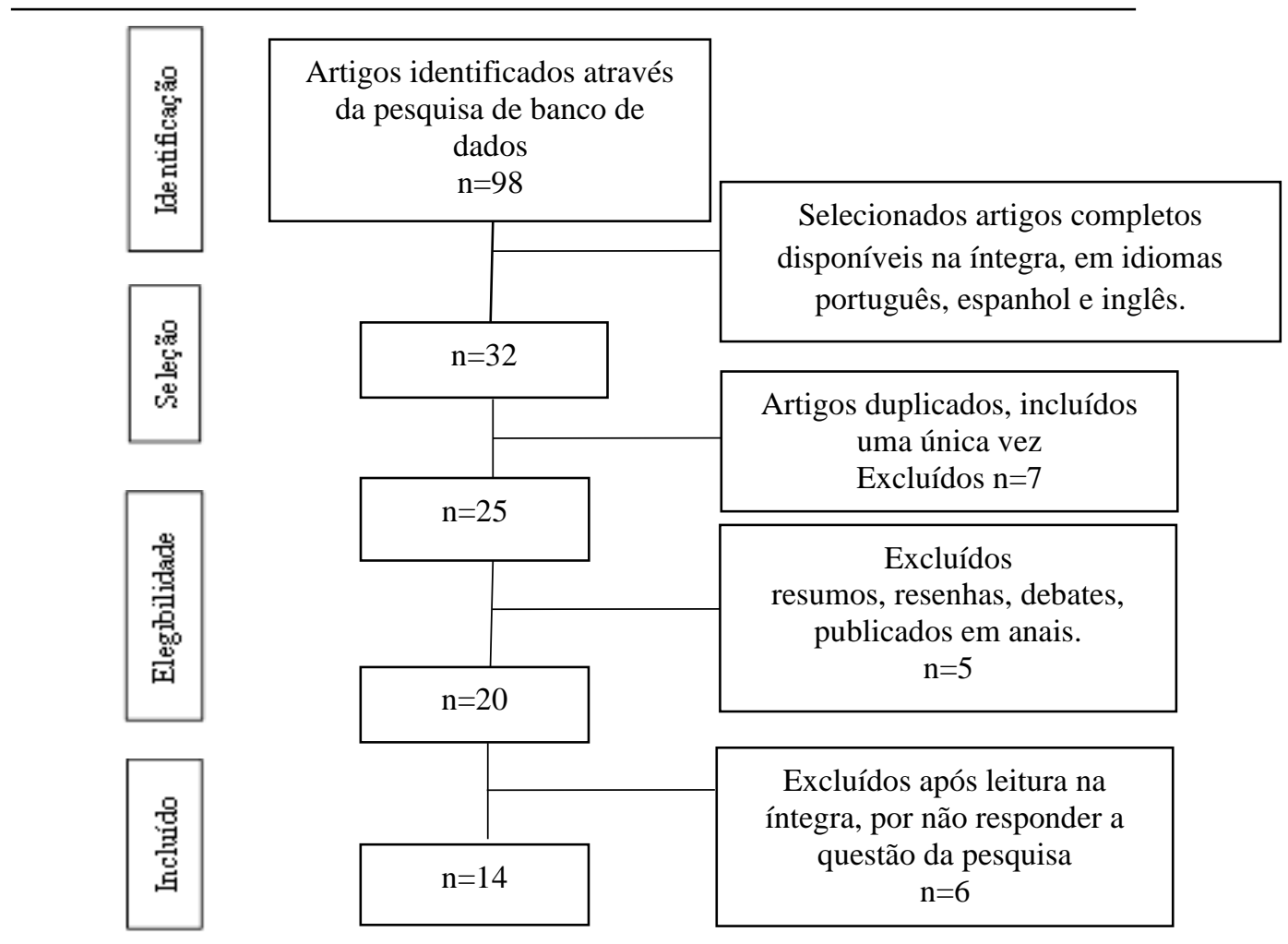

Fonte: Elaboração própria (2020).

No Fluxograma 1 observa-se que a partir da coleta de dados, localizaram-se 98 estudos que foram submetidos à primeira etapa de avaliação por meio da aplicação dos critérios de inclusão e exclusão. Assim, obteve-se uma amostra de 32 estudos ao final da primeira etapa de avaliação. Os artigos duplicados foram excluídos, resultando em 25 estudos.

Na segunda etapa, procedeu-se a leitura dos resumos dos 25 estudos para identificar aqueles que poderiam responder à questão da pesquisa. Desse processo, obteve-se uma amostra de 20 artigos. Após a leitura dos estudos na íntegra foram incluídos 14 estudos que puderam responder a questão de revisão.

O Quadro 1, a seguir, apresenta os artigos selecionados para o estudo de forma mais detalhada, com base no autor, título, ano e metodologia. 
Quadro 1. Caracterização dos artigos conforme autor, título, ano e metodologia.

\begin{tabular}{|c|c|c|c|}
\hline Autor & Título & Ano & Metodologia \\
\hline $\begin{array}{c}\text { Pereira, Passarin, Nishida, } \\
\text { \& Garcez. }\end{array}$ & $\begin{array}{c}\text { Meu Sonho É Ser Compreendido": } \\
\text { Uma Análise da Interação Médico- } \\
\text { Paciente Surdo durante Assistência à } \\
\text { Saúde. }\end{array}$ & 2020 & $\begin{array}{l}\text { Estudo observacional e } \\
\text { descritivo. }\end{array}$ \\
\hline $\begin{array}{c}\text { Cavagna, Silva, Braga \& } \\
\text { Andrade. }\end{array}$ & $\begin{array}{l}\text { O paciente surdo e suas vivências no } \\
\text { sistema de saúde: uma interface com a } \\
\text { enfermagem. }\end{array}$ & 2017 & $\begin{array}{c}\text { Estudo descritivo, de caráter } \\
\text { exploratório, } \\
\text { com abordagem qualitativa. }\end{array}$ \\
\hline Souza et al. & $\begin{array}{l}\text { Principais dificuldades e obstáculos } \\
\text { enfrentados pela comunidade surda no } \\
\text { acesso à saúde: uma revisão integrativa } \\
\text { de literatura }\end{array}$ & 2017 & $\begin{array}{l}\text { Revisão integrativa de } \\
\text { literatura. }\end{array}$ \\
\hline Reis \& Santos. & $\begin{array}{l}\text { Conhecimento e experiência de } \\
\text { profissionais das Equipes de Saúde da } \\
\text { Família no atendimento a pessoas } \\
\text { surdas. }\end{array}$ & 2019 & Estudo transversal. \\
\hline Abreu, Freitas, \& Rocha. & $\begin{array}{l}\text { A percepção dos surdos em relação ao } \\
\text { sistema de comunicação das unidades } \\
\text { de atenção primária à saúde. }\end{array}$ & 2015 & $\begin{array}{c}\text { Estudo descritivo de caráter } \\
\text { qualitativo. }\end{array}$ \\
\hline Pires \& Almeida. & $\begin{array}{l}\text { A percepção do surdo sobre o } \\
\text { atendimento nos serviços de saúde. }\end{array}$ & 2016 & $\begin{array}{c}\text { Estudo descritivo- } \\
\text { exploratório de abordagem } \\
\text { qualitativa. }\end{array}$ \\
\hline Lessa \& Andrade. & $\begin{array}{l}\text { Libras e o atendimento ao cliente surdo } \\
\text { no âmbito da saúde. }\end{array}$ & 2016 & $\begin{array}{l}\text { Pesquisa de campo } \\
\text { qualitativa. }\end{array}$ \\
\hline Yonemotu \& Vieira. & $\begin{array}{l}\text { Diversidade e comunicação: percepções } \\
\text { de surdos sobre atividade de educação } \\
\text { em saúde realizada por estudantes de } \\
\text { medicina }\end{array}$ & 2020 & Estudo qualitativo \\
\hline Sousa \& Almeida. & $\begin{array}{c}\text { Atendimento ao surdo na atenção } \\
\text { básica: perspectiva da equipe } \\
\text { multidisciplinar. } \\
\end{array}$ & 2017 & Revisão da literatura \\
\hline Dantas et al. & $\begin{array}{c}\text { Comunicação entre a equipe de } \\
\text { enfermagem e pessoas com deficiência } \\
\text { auditiva. }\end{array}$ & 2014 & $\begin{array}{c}\text { Estudo descritivo, } \\
\text { exploratório, com } \\
\text { abordagem qualitativa. }\end{array}$ \\
\hline $\begin{array}{l}\text { Soares, Lima, Santos \& } \\
\text { Ferreira. }\end{array}$ & $\begin{array}{l}\text { Como eu falo com você? a } \\
\text { comunicação do enfermeiro com o } \\
\text { usuário surdo. }\end{array}$ & 2018 & $\begin{array}{l}\text { Estudo descritivo, } \\
\text { exploratório, com } \\
\text { abordagem qualitativa. }\end{array}$ \\
\hline $\begin{array}{l}\text { Wetterich, Barroso \& } \\
\text { Freitas. }\end{array}$ & $\begin{array}{c}\text { A comunicação entre surdos e } \\
\text { profissionais da saúde: uma revisão } \\
\text { bibliográfica. }\end{array}$ & 2020 & Revisão bibliográfica. \\
\hline Saraiva et al. & $\begin{array}{l}\text { O silêncio das mãos na assistência aos } \\
\text { surdos nos serviços de saúde pública. }\end{array}$ & 2017 & Revisão de literatura. \\
\hline $\begin{array}{c}\text { Oliveira, Coura, Costa \& } \\
\text { França. }\end{array}$ & $\begin{array}{c}\text { Comunicação entre profissionais de } \\
\text { saúde-pessoas surdas: revisão } \\
\text { integrativa. }\end{array}$ & 2015 & $\begin{array}{l}\text { Revisão integrativa da } \\
\text { literatura. }\end{array}$ \\
\hline
\end{tabular}

Fonte: Dados da Pesquisa (2021).

A saúde é um direito do ser humano, assegurado na lei. Cabendo ao profissional de saúde se capacitar para promover a saúde da população (Cavagna et al., 2017). 
O principal desafio em atender o paciente surdo é devido à barreira comunicacional, devido à falta de preparo e conhecimento dos profissionais de saúde. Além do desafio linguístico, os surdos enfrentam obstáculos referentes à acessibilidade à saúde devido ao déficit de humanização na relação entre profissional e paciente, baixo conhecimento dos surdos sobre o processo de saúde-doença e ao difícil processo de inclusão destes na sociedade (Souza et al., 2017).

Segundo estudo de Reis e Santos (2019), dentre as barreiras de acessibilidade aos surdos, destacam-se: restrição de sua autonomia, privacidade comprometida e conflito ético devido a necessidade de intérprete familiar, ausência de intérprete profissional nas instituições de saúde, discriminação, preconceito, estigmas e estereótipos nos serviços de saúde, desatenção e inabilidade dos profissionais na busca de alternativas comunicacionais e atitudinais, falta de acolhimento e invisibilidade de suas necessidades singulares, dentre outros desafios.

De acordo com o estudo de Abreu et al. (2015) as principais dificuldades citadas no atendimento foram a falta de conhecimento de libras por parte dos profissionais de saúde, a falta de um intérprete no estabelecimento e a falta de paciência dos profissionais que prestam serviços de saúde.

As barreiras de comunicação, tanto do Surdo ao procurar os serviços de saúde, quanto dos profissionais que os atendem, são alarmantes. Quando não há comunicação efetiva, não há como contribuir em resolver os problemas do paciente (Pires \& Almeida, 2016).

$\mathrm{Na}$ área da saúde a comunicação é fundamental para suprir as necessidades dos deficientes auditivos, podendo ser feita através da escrita, leitura labial, e com a presença de um profissional para traduzir a língua brasileira para língua de sinais e vice-versa, ou pela utilização da Libras por profissionais da saúde (Lessa \& Andrade, 2016).

No estudo realizado por Cavagna et al. (2017), participantes da pesquisa apontam que na escrita encontra-se dificuldade de entendimento, devido a grafia dos profissionais e a língua portuguesa e que a utilização de gestos, mímicas, a fala lenta, também são utilizadas como estratégia de comunicação, porém, em algumas vezes, podem não demonstrar o entendimento esperado.

No estudo realizado por Pereira et al. (2020) demonstrou que a língua escrita pode ser uma barreira ao acesso dos surdos ao serviço de saúde, pois alguns apresentam dificuldades com a língua portuguesa, podendo ocasionar constrangimentos. Em situações em que se precisa detalhar a história, como em casos de violência, transtornos mentais ou questões que envolvem a sexualidade do paciente, observou-se que essa estratégia se mostra pouco eficaz, aumentando a possibilidade de erros de interpretação do caso.

Os surdos são considerados sujeitos passivos no processo de cuidado em saúde visto que têm pouca autonomia e responsabilidade nesse processo, pois, na maioria das vezes, precisam de um intérprete não tendo assim sua privacidade preservada (Yonemotu \& Vieira, 2020).

O acompanhante, na maioria das vezes, é quem explica ao profissional os problemas de saúde apresentados pelo surdo, afetando dessa forma a sua cidadania, por depender de outras pessoas para ter acesso às informações que poderiam melhorar a sua qualidade de vida (Sousa \& Almeida, 2017).

Abreu et al. (2015) afirma que com a presença do acompanhante, os surdos tem receio que sejam expostas questões confidenciais, em contrapartida sem a presença do mesmo, acabam privados de informações sobre decisões e condutas da assistência.

Segundo Pires e Almeida (2016) quando não há comunicação entre surdo-profissional o atendimento fica voltado para o acompanhante, prejudicando a interação do paciente com o profissional, provocando insegurança e constrangimento da parte do surdo. 
Devido a falta de comunicação dos profissionais, os surdos se sentem destratados, indignados, chateados e discriminados, já que o atendimento não supre suas necessidades. Isso faz com que muitos deles procurem as instituições de saúde apenas em extrema necessidade (Cavagna et al., 2017).

Ações e estratégias para a assistência aos usuários surdos deveriam ser de âmbito geral, orientadas por políticas públicas e planejadas pela gestão junto aos serviços de saúde. Porém, as ferramentas usadas para viabilizar a comunicação são individuais e pontuais, observa-se uma fragmentação das ações que não possibilitam a aquisição de novas práticas sólidas para promover a acessibilidade do portador de surdez (Soares et al., 2018).

Existe uma procura menor aos serviços de saúde pelos surdos, ficando assim mais vulneráveis ao surgimento de doenças que não foram detectadas precocemente. A falta de informação faz com que essa população acabe procurando respostas às suas dúvidas em fontes menos seguras, podendo causar problemas maiores devido à informação errada. Além disso, a busca por atendimentos de emergência é maior, assim como o número de internações hospitalares, se comparados ao público ouvinte (Yonemotu \& Vieira, 2020).

É fundamental a presença de um interprete para tradução e para participar ativamente de todo atendimento, a fim de tornar possível alcançar a compreensão (Pires \& Almeida, 2016).

A comunicação com deficientes auditivos é um grande obstáculo para os profissionais da saúde, pois prejudica o vínculo com esses pacientes e o atendimento que deve ser dispensado a eles. Reconhecer a deficiência na comunicação leva os profissionais a refletirem sobre essas questões e a melhorarem os serviços (Dantas et al., 2014).

Segundo Wetterich, Barroso e Freitas (2020), os profissionais da saúde relatam ter sentimentos de incapacidade, impotência, insegurança, constrangimentos, dentre outros, devido a dificuldade no processo de comunicação, e sentem necessidade de se capacitarem para proporcionarem um melhor atendimento ao surdo, com equidade e qualidade.

O profissional como prestador de serviços na área da saúde, deve zelar não somente por sua competência técnica, mas também pelo conhecimento aprofundado e por habilidades que favoreçam o estabelecimento de comunicação e relações de caráter interpessoal, visto que a barreira linguística pode gerar frustação e insegurança (Pereira et al., 2020).

A ausência de qualificação adequada para trabalhar com surdos contraria o Decreto ${ }^{\circ} 5.626 / 2005$, que regulamenta a inclusão plena dos surdos nos serviços de saúde, garantindo a atenção integral à saúde, por profissionais capacitados para o uso de libras e/ou para tradução ou interpretação para pessoas surdas ou aqueles não usuários da Língua Brasileira de Sinais, oferecendo ao surdo um atendimento de qualidade e acessível, garantido assim a universalidade e equidade da atenção (Reis \& Santos, 2019).

As barreiras de acesso impõem importantes constrangimentos que sinalizam o descuidado e/ou despreparo de profissionais no processo de trabalho, implicando, assim, em uma Atenção Primária à Saúde restritiva à diversidade de necessidades/demandas da população. É importante que o gestor público se comprometa com a formação permanente dos profissionais para lidarem adequadamente com demandas específicas como, por exemplo, de pessoas surdas (Reis \& Santos, 2019).

É preciso a tomada de providências efetivas para que os profissionais se comuniquem adequadamente com os clientes surdos, atuando com habilidades e atitudes adequadas a fornecer atendimento qualificado (Saraiva et al., 2017).

Curso de libras para profissionais de saúde é uma opção para tornar a comunicação eficaz com o paciente surdo e seus parentes. Quanto mais profissionais conseguirem aprender Libras, maior a possibilidade de respeito à inclusão social e à cultura do surdo (Soares et al., 2018).

A inclusão social do surdo depende do preparo da população para acolhê-lo e interagir de forma adequada. Proporcionar acessibilidade requer não apenas capacitação, mas também conscientização da demanda dos pacientes surdos e da importância do cuidado individualizado e humano (Pereira et al., 2020). 
Oliveira, Coura, Costa e França (2015) aponta que cabe aos profissionais da saúde refletir sobre a diversidade humana de forma que a barreira que os separa dos pacientes surdos seja reduzida e assim haja aceitação da língua e da forma de comunicação com os mesmos, conscientizando-se das implicações da surdez e superando os obstáculos para proporcionar um cuidado humanizado e integral aos deficientes auditivos.

\section{Conclusão}

Esse estudo é relevante à medida que proporciona conhecer as dificuldades encontradas na assistência à saúde a pessoa com surdez. Com o conhecimento adquirido através da revisão de literatura, observa-se que são amplas as dificuldades na assistência ao paciente surdo, entre elas a falta de comunicação foi descrita como sendo a principal, visto que a maioria dos profissionais não tem conhecimento da libras, além disso destacou-se a ausência de intérpretes nas instituições de saúde, falta de acolhimento, dentre outras barreiras que acabam dificultando o atendimento.

Ressalta-se a necessidade do profissional de saúde se capacitar para promover a saúde da população, não excluindo os surdos, visto que a saúde é um direito de todos. Contudo, para que isso aconteça se faz necessário o conhecimento da libras, através de treinamento e educação continuada.

Essa pesquisa estimula a produção de novas pesquisas de temática similar, para que se possa contribuir futuramente no melhor atendimento e abordagem dos pacientes surdos.

\section{Referências}

Abreu, J. C., Freitas, J. M. R., \& Rocha, L. L. V. (2015). A percepção dos surdos em relação ao sistema de comunicação das unidades de atenção primária à saúde. Brazilian journal of surgery and clinical research, 9(1), 06-11.

Aragão, J. S., França, I. S. X., Coura, A. S., Sousa, F. S., Batista, J. D. L., \& Magalhães, I. M. O. (2015). Um estudo da validade de conteúdo de sinais, sintomas e doenças/agravos em saúde expressos em LIBRAS. Revista Latino-Americana de Enfermagem, 23(6), $1014-1023$.

Brasil. Decreto $n^{\circ}$ 5.626, de 22 de dezembro de 2005. Regulamenta a Lei no 10.436, de 24de abril de 2002, que dispõe sobre a Língua Brasileira de SinaisLibras, e o art.18 da Lei n 10.098, de 19 de dezembro de 2000. Diário Oficial da União, Poder Executivo, Brasília, DF, Seção 1, 2005.

BRASIL. Lei $\mathrm{n}^{\circ}$. 10.098, de 19 de Dezembro de 2000. Estabelece normas gerais e critérios básicos para a promoção da acessibilidade das pessoas portadoras de deficiência ou com mobilidade reduzida, e dá outras providências. Diário Oficial da União, Poder Executivo, Brasília, DF. <https://www.presidencia.gov.br/ccivil_03/Leis/L10098.htm>.

Brasil. Lei n ${ }^{\circ} 10.436$, de 24 de abril de 2002. Dispõe sobre a Língua Brasileira de Sinais-Libras e dá outras providências. Diário Oficial da União, Poder Executivo, Brasília, DF, nº 79 , Seção 1, 2002.

Cavagna, V. M., Silva, W. P. J., Braga, A. L. S., \& Andrade, M. (2017). O paciente surdo e suas vivências no sistema de saúde: uma interface com a enfermagem. Revista Enfermagem Atual In Derme, 80(18), 33-39.

Dantas, T. R. A, Gomes, T. M., Costa, T. F., Azevedo, T. R., Brito, S. S., \& Costa, K. N. F. M. (2014). Comunicação entre a equipe de enfermagem e pessoas com deficiência auditiva. Revista Enfermagem UERJ, 22(2), 169-174.

França, E. G., Pontes, M. A., Costa, G. M. C., \& França, I. S. X. (2016). Dificuldades de profissionais na atenção à saúde da pessoa com surdez severa. Ciencia y enfermería, 22(3), 107-116.

Lessa, R. T. C., \& Andrade, E. G. S. (2016). Libras e o atendimento ao cliente surdo no âmbito da saúde. Rev. Cient. Sena Aires, 5(2), 95-104.

Oliveira, Y. C. A, Celino, S. D. M., França, I. S. X., Pagliuca, L. M. F., \& Costa, G. M. C. (2015). Conhecimento e fonte de informações de pessoas surdas sobre saúde e doença. Interface-Comunicação, Saúde, Educação, 19(54), 549-560.

Oliveira, Y. C. A., Coura, A. S., Costa, G. M. C., \& França, I. S. X. (2015). Comunicação entre profissionais de saúde-pessoas surdas: revisão integrativa. Revista de Enfermagem UFPE on line, 9(2), 957-964.

Pereira, A. A. C., Passarin, N. P., Nishida, F. S., \& Garcez, V. F. (2020). "Meu Sonho É Ser Compreendido": Uma Análise da Interação Médico-Paciente Surdo durante Assistência à Saúde. Revista Brasileira de Educação Médica, 44(4), e121.

Pires, H. F., \& Almeida, M. A. P. T. (2016). A percepção do surdo sobre o atendimento nos serviços de saúde. Revista Enfermagem Contemporânea, 5(1), 6877.

Reis, V. S. L., \& Santos, A. M. (2019). Conhecimento e experiência de profissionais das Equipes de Saúde da Família no atendimento a pessoas surdas. Revista CEFAC, 21(1), e5418. 
Research, Society and Development, v. 10, n. 2, e38910212372, 2021

(CC BY 4.0) | ISSN 2525-3409 | DOI: http://dx.doi.org/10.33448/rsd-v10i2.12372

Santos, W. R., Neves, A. G. A., Floriano, L. K. L., Gusmão, C. M. P., \& Oliveira, M. M. (2020). Inclusão do paciente surdo nos serviços de saúde no âmbito da atenção primária e suas interfaces com o cuidado de enfermagem. Caderno De Graduação - Ciências Biológicas e da Saúde - UNIT - ALAGOAS, 6(2), 7386.

Saraiva, F. J. C., Moura, R. S, Tavares, N. V. S., Junior, B. L, Santos, I. S., \& Santos, R. F. M.(2017). O silêncio das mãos na assistência aos surdos nos serviços de saúde pública. Olhares Plurais, 2(17), 94-104.

Soares, I. P., Lima, E. M. M., Santos, A. C. M., \& Ferreira, C. B. (2018). Como eu falo com você? a comunicação do enfermeiro com o usuário surdo. Rev baiana enferm. 32, e25978.

Sousa, E. M., \& Almeida, M. A. P. T. (2017). Atendimento ao surdo na atenção básica: perspectiva da equipe multidisciplinar. Id on Line Rev. Psic., 10(33), $72-82$.

Souza, M. F. N. S., Araújo, A. M. B., Sandes, L. F. F., Freitas, D. A., Soares, W. D., Vianna, R. S. M., \& Sousa, A. A. D. (2017). Principais dificuldades e obstáculos enfrentados pela comunidade surda no acesso à saúde: uma revisão integrativa de literatura. Revista CEFAC, 19(3), 395-405.

Wetterich, C. B., Barroso, H. C. S. M., \& Freitas, D. A. (2020). A comunicação entre surdos e profissionais da saúde: uma revisão bibliográfica. Revista Eletrônica Debates Em Educação Científica E Tecnológica, 4(1), 130-152.

Yonemotu, B. P. R., \& Vieira, C. M. (2020). Diversidade e comunicação: percepções de surdos sobre atividade de educação em saúde realizada por estudantes de medicina. Revista Eletrônica de Comunicação, Informação e Inovação em Saúde, 14(2), 401-414. 